\title{
Notes on the continental malacofauna of Rhodes, with two new species for the fauna of the island
}

\author{
Barna Páll-Gergely \& Zoltán Csabai \\ Department of General and Applied Ecology, University of Pécs, Ifjúság útja 6., H-7624 Pécs, Hungary; \\ e-mail: pallgergely2@gmail.com
}

\begin{abstract}
PÁll-Gergely B. \& Csabai Z., 2008: Notes on the continental malacofauna of Rhodes, with two new species for the fauna of the island. - Malacologica Bohemoslovaca, 7: 76-78. Online serial at $<\mathrm{http}: / /$ mollusca.sav.sk $>$ 23-Sep-2008.
\end{abstract}

\begin{abstract}
Data for 15 terrestrial and freshwater snail (Gastropoda) species are given from 35 localities on Rhodes Island. An invasive species, Haitia acuta (Draparnaud, 1805), and a species occurring in brackish waters, Ovatella firminii (Payraudeau, 1826) are new species and genus to the fauna of the island. This is the second record of $O$. firminii from Greece, which is interesting from another point of view; it was found in freshwater (not brackish) about $6 \mathrm{~km}$ from the sea.
\end{abstract}

Key words: Greece, Rhodes, Gastropoda, Ovatella firminii, faunistics

\section{Introduction}

The malacofauna of Rhodes (Greece) is relatively well known, but malacologists have mostly focused on the terrestrial snails (e.g. Frank 1997, MAASSEN 1981, PAGET 1976). During the collecting tour of the Aquatic Macroinvertebrates Research Group of University of Pécs, we visited 58 aquatic sampling sites, mainly rivers and streams in Rhodes, between 12 and 26 February 2007. The main goal of the field trip was to decipher the aquatic insect fauna of the island. As additional result terrestrial and freshwater snails were collected. The aim of this paper is to provide an overview of the terrestrial and freshwater snails collected by our research group.

\section{Material and methods}

Aquatic macroinvertebrates (including snail specimens) were captured by sweeping with a long handled pond net (mesh size $0.5 \mathrm{~mm}$ ) just above the substrate, on water surface, and among the submerged or emergent vegetation. Beyond netting, specimens were captured by manual singling from surface of submerged stones, woodstocks, etc. At some non-aquatic sampling sites additional collecting was made by manual singling terrestrial snail specimens. All captured specimens were preserved in small vials filled with $70 \%$ ethanol.

The nomenclature follows the Gastropoda chapter of Fauna Europaea webpage (BANK 2007). For the identification of the snails we used the above-mentioned literatures and the first author's comparative private collection. The Albinaria taxa living in Rhodes are sometimes hard to determinate. A. olivieri differs from $A$. brevicollis by the sculpture of the neck and the typical oval aperture. Shells and alcohol material are deposited in the private collection of Barna Páll-Gergely. The list of sampling sites which provided snail specimens [in alphabetic order, sorted by Municipalities of Rhodes, with accurate co-ordinates (WGS 84), collecting method, abbreviations of collectors and type of sampling] are given in Table 1. The numbers after the collection data indicates the total number of the collected specimens. Except for the clausiliids, all of the samples are in $70 \%$ ethanol.

\section{Results and discussion}

The main aim of present paper is to give faunistical data about malacofauna of the island Rhodes. So far two books and an article dealt with the snails of Rhodes. Other faunistical and taxonomic information can be found in literatures not directly about the island. The book of PAgET (1976) contains 45 species, MAASSEN (1981) reports 44 species form 28 localities. The book of FrANK (1997) is mainly about Helicidae s.1. species, and after a literature survey she mentions 54 taxa of the island Rhodes.

Our samplings resulted in the capture of 275 individuals of freshwater and terrestrial snails (142 terrestrial and 133 freshwater) belonging to 15 taxa (3 freshwater and 12 land snails). Because our work focused to freshwater samplings only 13 species previously known from Rhodes were found, but two further recently collected species proved to be new in the fauna of the island: Ovatella firmiini and Haitia acuta.

Until our samplings, Ovatella firminii (Fig. 1) was known only from Kephallinia from Greece (RÄHLE 1980), and from the neighbouring countries, Turkey (SснÜтт 2001) and Italy (Cossignani \& Cossignani 1995). One fresh shell has been collected from Rhodes in the Valley of Butterflies (Petaloudes). This occurrence is very interesting, because the species thought to live in brackish waters (SCHÜTT 2001), but this habitat is characterized by freshwater. We can not be sure about that single Ovatella shell is originated from freshwater, while the stream runs towards the sea, but the locality where this shell was found lies about $6 \mathrm{~km}$ from the sea. 
Table 1. List of localities where snail specimens were collected. (Abbreviations in the head: Lon. (N): longitude (N), Lat. (E): latitude (E), leg.: collectors; abbreviations in column "Method": KS: kick and sweep water netting, WN: water netting above the substrate and among the vegetation, MS: manual singling; abbreviations in column "leg.”: CsZ: Zoltán Csabai, KA: András Kálmán, KZ: Zoltán Kálmán, PZs: Zsuzsanna Pap, SN: Nándor Soós; abbreviations in column "Type”: A: Aquatic sampling, T: terrestrial sampling)

\begin{tabular}{|c|c|c|c|c|c|}
\hline Collecting sites & Lon. (N) & Lat. (E) & Method & leg. & Type \\
\hline \multicolumn{6}{|l|}{ Municipality of Afandou } \\
\hline Arhipoli: River Loutanis 2 & $36^{\circ} 16^{\prime} 03^{\prime \prime}$ & $28^{\circ} 04^{\prime} 08^{\prime \prime}$ & $\mathrm{KS}, \mathrm{MS}$ & CsZ-KA-KZ-SN & $\mathrm{A}$ \\
\hline Arhipoli: River Loutanis 3 & $36^{\circ} 15^{\prime} 59^{\prime \prime}$ & $28^{\circ} 03^{\prime} 30^{\prime \prime}$ & $\mathrm{KS}, \mathrm{MS}$ & CsZ-KA-PZs-SN & A \\
\hline Arhipoli: River Loutanis 4 & $36^{\circ} 15^{\prime} 34^{\prime \prime}$ & $28^{\circ} 06^{\prime} 01^{\prime \prime}$ & $\mathrm{KS}, \mathrm{MS}$ & CsZ-KA-KZ-SN & A \\
\hline Kolympia: River Loutanis 1 & $36^{\circ} 15^{\prime} 24^{\prime \prime}$ & $28^{\circ} 06^{\prime} 55^{\prime \prime}$ & $\mathrm{KS}, \mathrm{MS}$ & CsZ-KA-KZ-SN & $\mathrm{A}$ \\
\hline Kolympia: M. Tsampika, Panagia Tsampika & $36^{\circ} 14^{\prime} 10^{\prime \prime}$ & $28^{\circ} 09^{\prime} 01^{\prime \prime}$ & MS & KA-KZ-PZs-SN & $\mathrm{T}$ \\
\hline \multicolumn{6}{|l|}{ Municipality of Arhangelos } \\
\hline Arhangelos: stream 2 & $36^{\circ} 12^{\prime} 42^{\prime \prime}$ & $28^{\circ} 08^{\prime} 11^{\prime \prime}$ & $\mathrm{KS}, \mathrm{MS}$ & CsZ-KA-KZ-SN & A \\
\hline Arhangelos: stream 3 & $36^{\circ} 12^{\prime} 39^{\prime \prime}$ & $28^{\circ} 08^{\prime} 27^{\prime \prime}$ & $\mathrm{KS}, \mathrm{MS}$ & CsZ-KA-KZ-SN & A \\
\hline Charaki: River Gadouras & $36^{\circ} 09^{\prime} 23^{\prime \prime}$ & $28^{\circ} 03^{\prime} 53^{\prime \prime}$ & $\mathrm{KS}, \mathrm{MS}$ & CsZ-KA-KZ-SN & A \\
\hline Epta Piges (Seven Springs): lake 1 & $36^{\circ} 15^{\prime} 15^{\prime \prime}$ & $28^{\circ} 06^{\prime} 57^{\prime \prime}$ & $\mathrm{WN}, \mathrm{MS}$ & CsZ-KA-KZ-SN & $\mathrm{A}$ \\
\hline Epta Piges (Seven Springs): spring & $36^{\circ} 15^{\prime} 11^{\prime \prime}$ & $28^{\circ} 06^{\prime} 49^{\prime \prime}$ & $\mathrm{KS}, \mathrm{MS}$ & CsZ-KA-KZ-SN & $\mathrm{A}$ \\
\hline Epta Piges (Seven Springs): stream 1 & $36^{\circ} 15^{\prime} 13^{\prime \prime}$ & $28^{\circ} 06^{\prime} 48^{\prime \prime}$ & $\mathrm{KS}, \mathrm{MS}$ & CsZ-KA-KZ-SN & $\mathrm{A}$ \\
\hline Epta Piges (Seven Springs): stream 3 & $36^{\circ} 15^{\prime} 17^{\prime \prime}$ & $28^{\circ} 06^{\prime} 50^{\prime \prime}$ & $\mathrm{KS}, \mathrm{MS}$ & CsZ-KA-KZ-SN & $\mathrm{A}$ \\
\hline \multicolumn{6}{|l|}{ Municipality of Attavyros } \\
\hline Kritinia: Castle of Kritinia & $36^{\circ} 15^{\prime} 50^{\prime \prime}$ & $27^{\circ} 48^{\prime} 32^{\prime \prime}$ & MS & KA-KZ-PZs-SN & $\mathrm{T}$ \\
\hline Mandriko: River Mandriko & $36^{\circ} 17^{\prime} 28^{\prime \prime}$ & $27^{\circ} 51^{\prime} 24^{\prime \prime}$ & $\mathrm{KS}, \mathrm{MS}$ & CsZ-KA-KZ-SN & $\mathrm{A}$ \\
\hline \multicolumn{6}{|l|}{ Municipality of Kalithea } \\
\hline Faliraki: Olympia apartman & $36^{\circ} 21^{\prime} 14^{\prime \prime}$ & $28^{\circ} 12^{\prime} 11^{\prime \prime}$ & MS & KA-KZ-PZs-SN & $\mathrm{T}$ \\
\hline Psinthos: dry dam lake, stream & $36^{\circ} 18^{\prime} 43^{\prime \prime}$ & $28^{\circ} 05^{\prime} 50^{\prime \prime}$ & $\mathrm{KS}, \mathrm{MS}$ & CsZ-KA-KZ-SN & $\mathrm{A}$ \\
\hline \multicolumn{6}{|l|}{ Municipality of Kamiros } \\
\hline Apollona: River Gadouras 3 & $36^{\circ} 12^{\prime} 58^{\prime \prime}$ & $27^{\circ} 57^{\prime} 35^{\prime \prime}$ & $\mathrm{KS}, \mathrm{MS}$ & CsZ-KA-PZs-SN & A \\
\hline Apollona: River Gadouras 4 & $36^{\circ} 13^{\prime} 23^{\prime \prime}$ & $27^{\circ} 57^{\prime} 31^{\prime \prime}$ & $\mathrm{KS}, \mathrm{MS}$ & CsZ-KA-KZ-SN & $\mathrm{A}$ \\
\hline Apollona: stream & $36^{\circ} 11^{\prime} 13^{\prime \prime}$ & $27^{\circ} 57^{\prime} 31^{\prime \prime}$ & $\mathrm{KS}, \mathrm{MS}$ & CsZ-KA-KZ-SN & $\mathrm{A}$ \\
\hline Dimylia: River Platis & $36^{\circ} 17^{\prime} 37^{\prime \prime}$ & $28^{\circ} 00^{\prime} 23^{\prime \prime}$ & $\mathrm{KS}, \mathrm{MS}$ & CsZ-KA-KZ-SN & A \\
\hline Eleousa: stream & $36^{\circ} 16^{\prime} 17^{\prime \prime}$ & $28^{\circ} 02^{\prime} 23^{\prime \prime}$ & KS, MS & CsZ-KA-PZs-SN & $\mathrm{A}$ \\
\hline Salakos: River Argiros 1 & $36^{\circ} 18^{\prime} 43^{\prime \prime}$ & $27^{\circ} 56^{\prime} 19^{\prime \prime}$ & $\mathrm{KS}, \mathrm{MS}$ & CsZ-KA-KZ-SN & $\mathrm{A}$ \\
\hline Salakos: River Argiros 2 & $36^{\circ} 17^{\prime} 52^{\prime \prime}$ & $27^{\circ} 57^{\prime} 20^{\prime \prime}$ & $\mathrm{KS}, \mathrm{MS}$ & CsZ-KA-KZ-SN & $\mathrm{A}$ \\
\hline \multicolumn{6}{|l|}{ Municipality of Lindos } \\
\hline Lardos: River Kontari & $36^{\circ} 03^{\prime} 59^{\prime \prime}$ & $27^{\circ} 59^{\prime} 08^{\prime \prime}$ & $\mathrm{KS}, \mathrm{MS}$ & CsZ-KA-KZ-SN & $\mathrm{A}$ \\
\hline Lardos: River Lardos & $36^{\circ} 05^{\prime} 07^{\prime \prime}$ & $28^{\circ} 01^{\prime} 01^{\prime \prime}$ & $\mathrm{KS}, \mathrm{MS}$ & CsZ-KA-KZ-SN & $\mathrm{A}$ \\
\hline Lindos: Akropolis & $36^{\circ} 05^{\prime} 27^{\prime \prime}$ & $28^{\circ} 05^{\prime} 20^{\prime \prime}$ & MS & KA-SN & $\mathrm{T}$ \\
\hline \multicolumn{6}{|l|}{ Municipality of Petaloudes } \\
\hline Kremasti: River Kremastinos & $36^{\circ} 24^{\prime} 32^{\prime \prime}$ & $28^{\circ} 06^{\prime} 41^{\prime \prime}$ & $\mathrm{KS}, \mathrm{MS}$ & CsZ-KA-KZ-SN & $\mathrm{A}$ \\
\hline Petaloudes (Valley of butterflies): stream 2 & $36^{\circ} 20^{\prime} 05^{\prime \prime}$ & $28^{\circ} 03^{\prime} 47^{\prime \prime}$ & $\mathrm{KS}, \mathrm{MS}$ & CsZ-KA-KZ-SN & A \\
\hline Petaloudes (Valley of butterflies): stream 3 & $36^{\circ} 20^{\prime} 06^{\prime \prime}$ & $28^{\circ} 03^{\prime} 47^{\prime \prime}$ & $\mathrm{KS}, \mathrm{MS}$ & CsZ-KA-KZ-SN & $\mathrm{A}$ \\
\hline Petaloudes (Valley of butterflies): stream 4 & $36^{\circ} 20^{\prime} 07^{\prime \prime}$ & $28^{\circ} 03^{\prime} 47^{\prime \prime}$ & $\mathrm{KS}, \mathrm{MS}$ & CsZ-KA-KZ-SN & $\mathrm{A}$ \\
\hline Petaloudes (Valley of butterflies): stream 5 & $36^{\circ} 20^{\prime} 11^{\prime \prime}$ & $28^{\circ} 03^{\prime} 45^{\prime \prime}$ & $\mathrm{KS}, \mathrm{MS}$ & CsZ-KA-KZ-SN & $\mathrm{A}$ \\
\hline Petaloudes (Valley of butterflies): stream 7 & $36^{\circ} 20^{\prime} 15^{\prime \prime}$ & $28^{\circ} 03^{\prime} 42^{\prime \prime}$ & $\mathrm{KS}, \mathrm{MS}$ & CsZ-KA-KZ-SN & A \\
\hline Petaloudes (Valley of butterflies): stream 9 & $36^{\circ} 20^{\prime} 22^{\prime \prime}$ & $28^{\circ} 03^{\prime} 36^{\prime \prime}$ & $\mathrm{KS}, \mathrm{MS}$ & CsZ-KA-KZ-SN & $\mathrm{A}$ \\
\hline \multicolumn{6}{|l|}{ Municipality of Rhodes } \\
\hline Rhodes town: Rodini park, artificial fountain & $36^{\circ} 25^{\prime} 37^{\prime \prime}$ & $28^{\circ} 13^{\prime} 13^{\prime \prime}$ & $\mathrm{WN}, \mathrm{MS}$ & CsZ-KA-KZ-SN & $\mathrm{A}$ \\
\hline Rhodes town: Rodini park, stream & $36^{\circ} 25^{\prime} 36^{\prime \prime}$ & $28^{\circ} 13^{\prime} 08^{\prime \prime}$ & $\mathrm{KS}, \mathrm{MS}$ & CsZ-KA-KZ-SN & $\mathrm{A}$ \\
\hline Rhodes town: Old town, dry castel moat & $36^{\circ} 26^{\prime} 43^{\prime \prime}$ & $28^{\circ} 13^{\prime} 20^{\prime \prime}$ & MS & KA-KZ-PZs-SN & $\mathrm{T}$ \\
\hline Rhodes town: Monte Smith, Akropolis & $36^{\circ} 26^{\prime} 24^{\prime \prime}$ & $28^{\circ} 12^{\prime} 38^{\prime \prime}$ & MS & KA-KZ-PZs-SN & $\mathrm{T}$ \\
\hline \multicolumn{6}{|l|}{ Municipality of South Rhodes } \\
\hline Apolakkia: River Apolakkias & $36^{\circ} 05^{\prime} 09^{\prime \prime}$ & $27^{\circ} 47^{\prime} 48^{\prime \prime}$ & $\mathrm{KS}, \mathrm{MS}$ & CsZ-KA-KZ-SN & $\mathrm{A}$ \\
\hline
\end{tabular}

Haitia acuta is also new to the fauna of Rhodes (see. FRANK 1997). The origin of this species is North-America, but this invasive species is now widely distributed in almost all European countries (ANDERSON 2003). Both are new species and genera to the fauna of the island.

Three species of freshwater snails were found, the most common being Melanopsis buccinoidea and Galba truncatula. The first species mainly occurs in oxygen-rich running waters, while the second one prefers mainly warm and shallow standing waters (lakes, ponds) and slow flowing rivers. Galba truncatula tolerates lower oxygen levels (personal observations). The two species have not been found sympatrically in waterbodies of Rhodes.

\section{Collected taxa with localities}

The data are listed in order: locality, date of collection, the number of found specimens.

Melanopsis buccinoidea (Olivier, 1801) - Apollona: River Gadouras 3, 22 Feb 2007, 2; Arhangelos: stream 2, 24 Feb 2007, 2; Arhangelos: stream 3, 24 Feb 2007, 3; Arhipoli: River Loutanis 4, 19 Feb 2007, 6; Epta Piges 


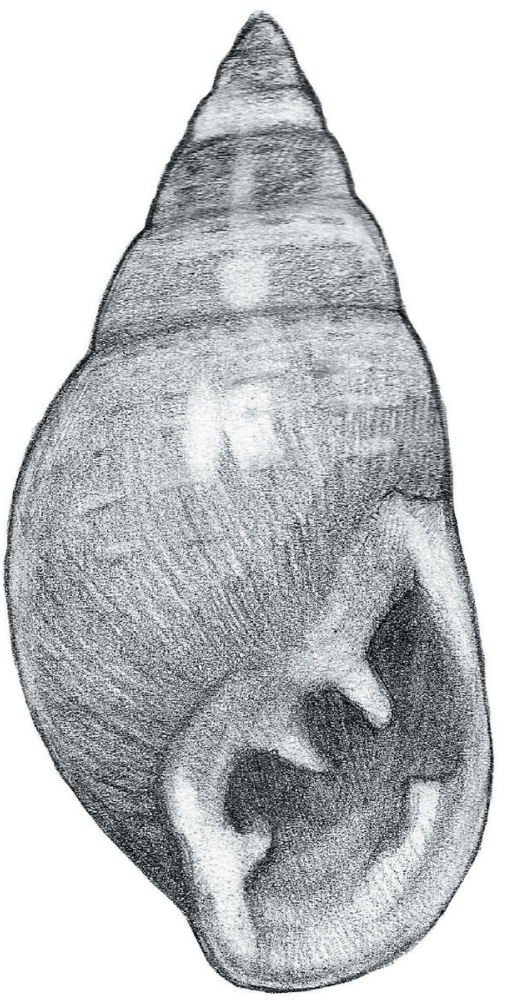

Fig. 1. Habitus of the shell of Ovatella firminii, a new species and genus to the fauna of Rhodes (Greece, Rhodes, Petaloudes; Valley of butterflies 7., original drawing by Csaba Horváth). Actual height: $8.3 \mathrm{~mm}$.

(Seven Springs): spring, 14 Feb 2007, 8; Epta Piges (Seven Springs): stream 1, 14 Feb 2007, 2; Epta Piges (Seven Springs): stream 3, 15 Feb 2007, 1; Kolympia: River Loutanis 1, 19 Feb 2007, 21; Kremasti: River Kremastinos, 21 Feb 2007, 1; Petaloudes (Valley of butterflies): stream 2, 21 Feb 2007, 7; Petaloudes (Valley of butterflies): stream 3, $21 \mathrm{Feb}$ 2007, 2; Petaloudes (Valley of butterflies): stream 4, 21 Feb 2007, 2; Petaloudes (Valley of butterflies): stream 5, 21 Feb 2007, 2; Petaloudes (Valley of butterflies): stream 7, 21 Feb 2007, 2; Petaloudes (Valley of butterflies): stream 9, 21 Feb 2007, 2; Psinthos: dry dam lake, stream, 25 Feb 2007, 2; Rhodes town: Rodini park, stream, 23 Feb 2007, 5 .

Galba truncatula (O.F. Müller, 1774) - Apolakkia: River Apolakkias, 16 Feb 2007, 8; Arhipoli: River Loutanis 2, 19 Feb 2007, 5; Arhipoli: River Loutanis 3, 22 Feb 2007, 23; Arhipoli: stream, 19 Feb 2007, 1; Eleousa: stream, 22 Feb 2007, 2; Epta Piges (Seven Springs): lake 1, 14 Feb 2007, 5; Mandriko: River Mandriko, 20 Feb 2007, 1; Rhodes town: Rodini park, artificial fountain, $23 \mathrm{Feb}$ 2007, 1; Salakos: River Argiros 1, 20 Feb 2007, 5; Salakos: River Argiros 2, 20 Feb 2007, 4.

Ovatella firminii (Payraudeau, 1826) - Petaloudes (Valley of butterflies): stream 7, $21 \mathrm{Feb} 2007,1$.

Haitia acuta (Draparnaud, 1805) - Rhodes town: Rodini park, artificial fountain, 23 Feb 2007, 6; Rhodes town: Rodini park, stream, 23 Feb 2007, 2.

Orculella ignorata Hausdorf, 1996 - Charaki: River Gadouras, 24 Feb 2007, 1.
Mastus emarginatus turgidus (Kobelt, 1877)- Kolympia: M. Tsampika, Panagia Tsampika, 19 Feb 2007, 1; Lardos: River Kontari, 17 Feb 2007, 1.

Zebrina fasciolata (Olivier, 1801) - Faliraki: Olympia apartman, 16 Feb 2007, 12.

Rumina decollata (Linnaeus, 1758) - Rhodes town: Old town, dry castel moat, $13 \mathrm{Feb} 2007,2$.

Albinaria brevicollis koskinensis (Pollonera, 1916) - Kolympia: M. Tsampika, Panagia Tsampika, 19 Feb 2007, 10; Lindos: Akropolis, 24 Feb 2007, 25.

Albinaria klemmi Paget, 1971 - Kolympia: M. Tsampika, Panagia Tsampika, 19 Feb 2007, 2.

Albinaria olivieri (Roth, 1839) - Rhodes town: Monte Smith, Akropolis, 13 Feb 2007, 20; Rhodes town: Old town, dry castel moat, $13 \mathrm{Feb} 2007,41$; Rhodes town: Rodini park, stream, 23 Feb 2007, 2.

Cochlicella acuta (O.F. Müller, 1774) - Lardos: River Lardos, 17 Feb 2007, 19.

Metafruticicola pellita pellita (J. Fèrussac, 1819) - Rhodes town: Old town, dry castel moat, 13 Feb 2007, 1.

Metafruticicola pellita depressa (Kobelt, 1904) - Kritinia: Castle of Kritinia, $20 \mathrm{Feb} 2007,1$.

Eobania vermiculata (O.F. Müller, 1774) - Apolakkia: River Apolakkias, 16 Feb 2007, 1; Kolympia: M. Tsampika, Panagia Tsampika, 19 Feb 2007, 2.

\section{Acknowledgments}

Authors' thanks are due to Ruud A. Bank (Hoofddorp) for sending the actualized checklist of the Greek continental malacofauna (www.faunaeur.org), and for updating the English, to Csaba Horváth (Bátonyterenye) for the drawing of $O$. firminii and to Péter Sólymos (Budapest) for his help in several ways. Thanks also for the valuable and constructive comments of three anonymous reviewers. This study was financially supported by the Student Union of Faculty of Sciences, University of Pécs (Pécs, Hungary) and Post-Bau ltd. (Pécs, Hungary).

\section{References}

Anderson R., 2003: Physella (Costatella) acuta Draparnaud in Britain and Ireland - its taxonomy, origins and relationships to other introduced Physidae. - Journal of Conchology, 38 (1): 7. BANK R.A., 2007: Fauna Europaea: Gastropoda. - Fauna Europaea version 1.3, http://www.faunaeur.org.

Cossignani T. \& Cossignani V., 1995: Atlante delle conchiglie terrestri e dulciacquicole italiane. - L'informatiore Piceno, Ancona, pp. 48-49.

Frank C., 1997: Die Molluskenfauna der Insel Rhodos, 2. Teil. - Stapfia, 48: 1-179.

MaAssen W.J.M., 1981: De Molluskenfauna van het griekse eiland Rhodos. - De Kreukel, 17: 21-32.

Paget O.E., 1976: Die Molluskenfauna der Insel Rhodos. 1. Teil. - Annalen des Naturhistorischen Museums in Wien, 80: 681-780.

RäHLE W., 1980: Land- und Süsswassermollusken von Kephallinia und Zakynthos (Ionische Inseln). - Archiv für Molluskenkunde, 110 (4-6): 199-224.

SснÜтт H., 2001: Die Türkischen Landschnecken 1758-2000. 3. Vollständig revidierte und erweiterte auflage. - Acta Biologica Benrodis, Supplementband 4, 549 pp. 